\title{
Integrated and Independent Learning of Hand-Related Constituent Sequences
}

\author{
Michael P. Berner and Joachim Hoffmann \\ University of Würzburg
}

\begin{abstract}
In almost all daily activities fingers of both hands are used in coordinated succession. The present experiments explored whether learning in such tasks pertains not only to the overall sequence spanning both hands but also to the constituent sequences of each hand. In a serial reaction time task, 2 repeating hand-related sequences were intertwined, so that actions of one hand alternated with actions of the other hand. Integrated learning of the overall sequence was weak when the constituent sequences were uncorrelated (Experiment 1) and massive when they were correlated (Experiment 2). Both experiments yielded evidence suggesting partly independent learning of the hand-related sequences. There were no reliable indications of intermanual transfer of this hand-related sequence knowledge. The findings suggest that after sufficient training of coordinated action sequences involving several limbs, a part of the acquired sequence knowledge begins to be represented in an effector-specific manner.
\end{abstract}

Keywords: sequence learning, serial reaction time (SRT) task, intermanual transfer, effector specificity

In many everyday tasks we have to coordinate action sequences of the two hands or other effectors. For example, buttoning a shirt or folding a piece of paper requires the use of both hands in a fixed sequence of coordinated actions. Typically, we are oblivious to the involvement of both hands in such activities unless one of the involved effectors becomes impaired, for example, as a result of an injury. Although learning of action sequences has long been the subject of theorizing and research (e.g., Lashley, 1951; for reviews, see, e.g., Clegg, DiGirolamo, \& Keele, 1998; Hoffmann, 2001; Rhodes, Bullock, Verwey, Averbeck, \& Page, 2004), this multieffector aspect of sequential performance has received virtually no attention.

This is quite perplexing, given that one of the preferred methods of investigating sequence learning, the serial reaction time (SRT) task as introduced by Nissen and Bullemer (1987), usually requires responses with fingers of both hands. In a typical SRT task, participants respond to stimuli by pressing respectively assigned response keys. Each response triggers the presentation of the next stimulus, which in turn prompts the next response and so on. In this way, a sequence of stimuli is accompanied by a sequence of responses. Usually, stimuli follow each other in a redundant or structured way, often in a fixed sequence. Accordingly, the required keypresses also follow a redundant or fixed sequence.

Michael P. Berner and Joachim Hoffmann, Department of Psychology, University of Würzburg, Würzburg, Germany.

This research was supported by German Research Council Grants HO 1301/12-1 and HO 1301/12-2 (awarded to Joachim Hoffmann). We thank Charles Shea and Luis Jiménez for helpful comments on earlier versions of this article. We thank Marjorie Kinney for improving the English of an earlier version of this article.

Correspondence concerning this article should be addressed to Michael P. Berner, Institut Psychologie III, Universität Würzburg, Röntgenring 11, Würzburg D-97070, Germany. E-mail: berner@psychologie uni-wuerzburg.de
Learning of the implemented sequential structure is assessed after some practice by replacing the structured sequence with a random sequence. A decrement in performance in such a test block indicates sequence learning.

As already noted, responding in SRT tasks almost always involves fingers of both hands, so that the overall sequence is composed of two hand-related sequences. Imagine, for example, that the keys $1,2,3$, and 4 are to be pressed with the middle and index finger of the left hand and the index and middle finger of the right hand, respectively. Under these conditions, the repeating overall sequence 13423124 is composed of two intertwined, handrelated sequences: $1 \_2 \_12$ for the left hand and 34 _ $3 \_4$ for the right hand. In that sense, the SRT task provides a model not only for learning an overall sequence but also for learning the interwoven hand-related sequences. However, to the best of our knowledge, previous studies employing the SRT task have focused exclusively on integrated learning of an overall sequence spanning both hands. The possibility that at least partly independent learning of the hand-related constituent subsequences might occur has never been examined.

Note that the question of whether hand-related sequences might be learned at least partly independently of one another is to be treated separately from the related question regarding the representational nature of acquired independent sequence knowledge. This latter question specifically concerns whether independent knowledge about hand-related sequences is represented in a handspecific manner, so that it is not transferable from the left hand to the right hand or vice versa. Next, we summarize research relevant to each of these two issues in turn.

\section{Independent Versus Integrated Sequence Learning}

Previous investigations into independent versus integrated learning of two concurrent sequences were focused predominantly on combining a response-relevant stimulus sequence with another 
response-irrelevant stimulus sequence (Deroost \& Soetens, 2006; Mayr, 1996; Riedel \& Burton, 2006; Rüsseler, Münte, \& Rösler, 2002; see also Deroost, Zeischka, \& Soetens, 2008). For example, participants in Riedel and Burton's (2006) experiment listened to a list of words. There were four possible words and four possible voices. Presentation was arranged such that the words appeared in a repeating sequence independently of an uncorrelated sequence of voices. Participants were instructed to respond either to the identity of the word or to the identity of the voice; the respectively other stimulus attribute was response irrelevant. This experiment and others (Deroost \& Soetens, 2006; Rüsseler et al., 2002) consistently found evidence for learning of the response-relevant sequence, whereas learning of the response-irrelevant sequence has proven elusive.

These experiments do not rule out the possibility that two concurrent sequences might be learned independently of one another when each is implemented in response-relevant stimuli. However, evidence for this is relatively scarce (Berner \& Hoffmann, 2008; Frensch \& Miner, 1995; Mayr, 1996; Schmidtke \& Heuer, 1997). On each trial in Mayr's (1996) Experiment 1, one of four symbols was presented in one of four locations. Participants responded to the symbols, which appeared in a repeating sequence, by pressing respectively assigned keys. At the same time, the locations were determined by another repeating sequence. Inasmuch as it was necessary for participants to move their eyes from the previous location to the current location in the location sequence in order to be able to recognize the current symbol, the location sequence can be considered as requiring responses as well (cf. Stadler, 1989; see also Koch \& Hoffmann, 2000a; sequence learning for eye movement responses has indeed been demonstrated; see Kinder, Rolfs, \& Kliegl, 2008; Marcus, Karatekin, \& Markiewicz, 2006). At the end of the experiment, test blocks were implemented in which either only one of the sequences was replaced with a random sequence or both of the sequences were randomized. Participants' RTs increased compared with adjacent regular blocks in all of these test blocks, but the RT increase was more pronounced when both sequences were randomized in the same test block than when only one of the sequences was randomized. This pattern of results suggests that the symbol (keypress) sequence and the location (eye movement) sequence had been learned independently of one another. ${ }^{1}$

Schmidtke and Heuer's (1997) participants responded alternately to (a) a repeating sequence of visual stimuli with manual keypresses and (b) a repeating sequence of tones by pressing a foot pedal. In one condition, the visual (key) sequence and the auditory (pedal) sequence followed different fixed six-element sequences (i.e., correlated sequences). After some practice, the two sequences were shifted relative to each other, so that only regularities between them were altered whereas the within-sequence regularities remained unaffected (shift probe). Nevertheless, both the manual and the foot responses were delayed, which indicated that participants had learned the interrelations between both sequences. In additional test blocks, either the visual (key) sequence was replaced with a random sequence while the auditory (pedal) sequence remained intact or vice versa. In these random probes, significant RT increases were observed for both tasks, even for that task for which the sequence remained intact. Both findings indicate integrated learning of the two sequences. However, as Keele, Ivry, Mayr, Hazeltine, and Heuer (2003, p. 323) have pointed out, one can conclude from the finding that RTs increased more in the random probes than in the shift probe that some independent learning of the two sequences occurred in addition to the integrated learning of the compound sequence.

Berner and Hoffmann (2008) investigated concurrent learning of two response-relevant hand-related sequences in a bimanualbisequential variant of the SRT task. On every trial participants responded simultaneously to the positions of stimuli by pressing the corresponding pair of keys with the appropriate fingers of the left and the right hand, respectively. The stimuli for the left-hand side and those for the right-hand side followed different, uncorrelated sequences. After extensive practice, independent learning was assessed by replacing either one or both of the hand-related sequences with a random sequence. Participants' performance in terms of RT suffered significantly more when both sequences were randomized than when only one of the sequences was randomized. Furthermore, left-hand errors increased only when the left-hand sequence was abolished; right-hand errors increased only when the right-hand sequence was abolished. This pattern of results indicates independent learning of the two hand-related sequences.

Taken together, the findings just reviewed suggest that when two response-related sequences are interwoven, sequence learning occurs not only with regard to the overall sequence but also with regard to the constituent sequences. However, it remains unclear whether the acquired hand-related sequence knowledge pertains to stimuli or responses (cf. Hoffmann, Martin, \& Schilling, 2003; Hoffmann \& Sebald, 1996; Nattkemper \& Prinz, 1997). For example, participants in Schmidtke and Heuer's (1997) experiment might have learned independently about the sequence of visual stimuli and the sequence of acoustic stimuli or about the sequence of hand movements and the sequence of foot movements. If independent learning of concurrent sequences performed with different effectors indeed pertains to responses, a related issue is whether the acquired sequence knowledge can be transferred to other effectors or not.

\section{Effector-Specific Versus Effector-Independent Sequence Learning}

Results from numerous experiments indicate that knowledge acquired about a sequence of actions to be performed with one hand is easily transferred to the other hand (e.g., Deroost, Zeeuws, \& Soetens, 2006; Grafton, Hazeltine, \& Ivry, 2002; Japikse, Negash, Howard, \& Howard, 2003; Panzer et al., 2007; see also Cohen, Ivry, \& Keele, 1990, Experiment 2; Grafton, Hazeltine, \& Ivry, 1998; Keele, Jennings, Jones, Caulton, \& Cohen, 1995; Willingham, Wells, Farrell, \& Stemwedel, 2000, Experiment 2). Indeed, sequence knowledge has been shown to be represented in multiple formats (cf. Clegg et al., 1998) that lend themselves to intermanual transfer. For example, sequence knowledge can pertain to sequences of response effects (e.g., Hazeltine, 2002; Hoffmann, Sebald, \& Stöcker, 2001; Stöcker \& Hoffmann, 2004;

\footnotetext{
${ }^{1}$ However, no such learning of a location (eye movement) sequence in addition to a separate keypress sequence was evident in the experiments of Deroost and Soetens (2006; Experiment 1) or Rüsseler et al. (2002). Thus, the putative equivalence between eye movements and more overt and effortful responses, such as keypressing, appears to be questionable, at least when it comes to independent learning of concurrent sequences.
} 
Stöcker, Sebald, \& Hoffmann, 2003; Ziessler, 1998; Ziessler \& Nattkemper, 2001) and sequences of stimuli (e.g., Clegg, 2005; Frensch \& Miner, 1995; Remillard, 2003) as well as to relational patterns between consecutive responses (e.g., Hoffmann \& Koch, 1998; Hoffmann \& Sebald, 1996; Koch \& Hoffmann, 2000a, 2000 b) or to sequences of response locations (Willingham et al., 2000; see also Willingham, 1999; Witt \& Willingham, 2006). All of these different sequence representations are independent of the hand used for responding, so that sequence knowledge acquired during practice with one hand can be used just as well when responding with the other hand.

Such transfer of sequence learning can be nicely accounted for by modular theories of sequence representation (e.g., Keele et al., 1995), which hold that sequence information is represented in a relatively abstract manner in a module that can be interfaced with various effector systems. The specific motor commands required for executing a particular sequence of actions are assumed to be computed in a separate module in which no knowledge about the sequence would be represented. A number of studies have reported successful rescaling of sequence structure (i.e., the way in which the sequence is partitioned into chunks) in amplitude, force, or time that is consistent with this conceptualization, at least when rescaling is proportional across the entire sequence (e.g., Dean, Kovacs, \& Shea, 2008; Muehlbauer, Panzer, \& Shea, 2007; Wilde \& Shea, 2006).

Recently, however, a number of experiments have demonstrated an effector-specific component of sequencing skill following extensive practice of the same, deterministic sequence of movements (Verwey \& Clegg, 2005; see also Bapi, Doya, \& Harner, 2000; Berner \& Hoffmann, 2008, 2009; Jordan, 1995; Park \& Shea, 2003, 2005; Verwey \& Wright, 2004). For example, Verwey and Clegg (2005, Experiment 1) had their participants complete more than 1,000 repetitions of a sequence of keypresses with the fingers of one hand before having them switch to responding with the other hand. Participants were significantly faster when responding with the transfer hand to the practiced sequence than when responding to a new unpracticed sequence and thus showed considerable intermanual transfer. However, responses with the transfer hand to the practiced sequence were not as fast as with the practiced hand, and this indicated a nontransferable component of sequence knowledge.

Such findings of a nontransferable component of sequence learning are consistent with a model formulated by Hikosaka et al. (1999; see also Hikosaka, Nakamura, Sakai, \& Nakahara, 2002). Building on findings from neuroscience and behavioral research, they proposed that sequence learning occurs in parallel on two independent levels, more specifically, in two separate cortical systems. Sequence learning in terms of visual-spatial coordinates is assumed to be dominant early in the learning process, and sequence knowledge acquired in this way is effector-independent. In contrast, sequence leaning in terms of motor coordinates (e.g., muscle activations, joint angles) is assumed to become prominent at later stages in learning and to result in effector-specific, nontransferable sequence knowledge.

To the best of our knowledge, almost all demonstrations of the acquisition of effector-specific sequence knowledge have so far investigated learning of sequential actions involving a single effector. Only Berner and Hoffmann (2008) have investigated effector-specific sequence learning for multieffector sequential action in a bimanual-bisequential SRT task, as described above. They found evidence for a transferable as well as for a nontransferable component of independently acquired knowledge about the hand-related sequences.

\section{The Current Study}

The present set of experiments was designed to determine whether independent learning of hand-related sequences as reported by Berner and Hoffmann (2008) for simultaneous bimanual responses persists under conditions of alternating stimulus presentation and alternating responses. Under these conditions, the stimuli/responses belonging to a hand-related sequence are separated by intervening stimuli/responses belonging to the other handrelated sequence. This might favor integrated sequence learning, because associations between subsequent sequence elements will be associations across hands rather than associations within hands. For example, sequence learning in terms of to-be-generated response effects (e.g., Hoffmann et al., 2001; Ziessler, 1998; Ziessler $\&$ Nattkemper, 2001) will rely on associations between a response executed with one hand and the next imperative stimulus for the other hand. The occurrence of independent learning of effectorrelated sequences was tested not only for sequences of different length (Experiment 1) but also for hand-related sequences of the same length (Experiment 2), so that the two sequences are highly correlated. Such correlation is probably more representative of everyday sequential actions and might additionally favor the acquisition of integrated sequence learning. Also, we implemented tests of intermanual transfer in order to discover whether any independently acquired sequence knowledge is effector-specific or is available for intermanual transfer.

\section{Experiment 1}

On each trial in Experiment 1, participants responded to an imperative stimulus appearing in one of eight vertically aligned locations on the monitor by pressing the corresponding key. The four topmost keys were to be pressed with fingers of the left (right) hand, and the four bottommost keys were to be pressed with fingers of the right (left) hand. As far as these aspects of the setting are concerned, this task is isomorphic to a standard SRT task. As an additional constraint, the imperative stimulus appeared alternately in the left-hand and right-hand halves of the display. The stimuli in the left-hand half followed a fixed repeating sequence independent of and different from that for the stimuli in the right-hand half. These two interleaved sequences were not equal in length, so that together they established a compound sequence considerably longer and more complex than its constituent sequences.

To the extent that the two hand-related sequences are learned independently of one another, randomizing one of them while retaining the other should incur costs (i.e., an increase in RT and error rate) for the hand that loses its sequence but not for the other hand. If independently acquired sequence knowledge is represented in an effector-independent (i.e., transferable) manner, transferring one hand-related sequence from the practiced hand to the other hand should not incur costs for that other hand. To the extent that learning is integrated, there cannot be any intermanual transfer. 


\section{Method}

Participants. A total of 24 individuals (mean age $=24$ years) volunteered to participate in Experiment 1 in partial fulfillment of course requirements or for payment of 15 euros. Due to experimenter error, handedness information was not obtained from 1 participant. Of the remaining participants, 21 were predominantly right-handed, 1 was ambidextrous, and 1 was predominantly lefthanded.

Task and design. The presence or absence of the hand-related sequences constituted the within-subjects factor. Participants practiced both sequences extensively (for 46 blocks) before several test blocks were inserted between the regular sequence blocks. In particular, the following types of test blocks were implemented (see also Table 1): The left-hand sequence was replaced with a pseudorandom sequence while the right-hand sequence was retained (test block $L_{\text {rand }}$ ), or the right-hand sequence was replaced with a pseudorandom sequence while the left-hand sequence was retained $\left(R_{\text {rand }}\right)$, or both hand-related sequences were replaced with pseudorandom sequences in the same test block $\left(L R_{\text {rand }}\right)$. In addition to these randomization probes, there were test blocks in which either the sequence practiced with the left hand was transferred to the right hand while a pseudorandom sequence was presented for the left hand $\left(L_{\text {trans }}\right)$ or the sequence practiced with the right hand was transferred to the left hand while a pseudorandom sequence was presented for the right hand $\left(R_{\text {trans }}\right)$. In these transfer probes, the sequences were transferred from the one hand to the other in such a way that the top-to-bottom ordering of stimulus (and key) locations was maintained (i.e., the sequences were not mirrored). The vertical alignment of response keys ensured that, for both hands, corresponding keys were operated with homologous fingers. Thus, the transfer conditions conserved the practiced sequence not only in terms of visual-spatial coordinates but also in terms of the effectors themselves.

Apparatus and materials. Stimulus presentation and response registration were controlled by the E-Prime software package (Schneider, Eschman, \& Zuccolotto, 2002). When responding, participants used eight vertically aligned keys that were mounted on the far side of a stick, so that participants depressed the keys toward their body (see Figure 1). The stick was attached perpendicularly to the tabletop. The keys were connected to the serial port of the computer via the Psychology Software Tools' Serial Response Box Model 200a. An asterisk appeared in black on a white

Table 1

Assignment of Fixed Sequences and Pseudorandom Sequences to the Left and the Right Hand in Regular Blocks and in the Different Types of Test Blocks

\begin{tabular}{lll}
\hline & \multicolumn{2}{c}{ Sequence } \\
\cline { 2 - 3 } Block & Left hand & Right hand \\
\hline $\begin{array}{l}\text { Regular block } \\
\text { Test block }\end{array}$ & practiced left & practiced right \\
$L_{\text {rand }}$ & random & practiced right \\
$R_{\text {rand }}$ & practiced left & random \\
$L R_{\text {rand }}$ & random & random \\
$L_{\text {trans }}$ & random & practiced left \\
$R_{\text {trans }}$ & practiced right & random \\
\hline
\end{tabular}

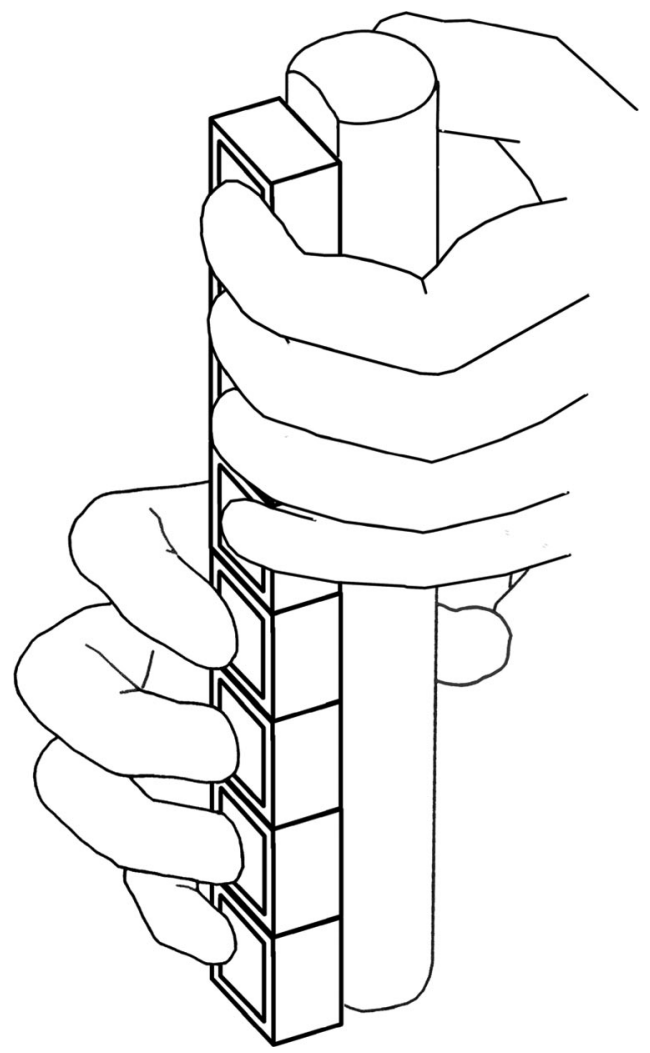

Figure 1. Illustration of the response key setup employed in Experiment 1.

background in any one of eight vertically aligned locations on the screen; each of these locations was marked by a black square (side length $=14 \mathrm{~mm}$ ). There was a distance of $4 \mathrm{~mm}$ between any two adjacent squares. The keys were assigned compatibly to the squares (locations) on the screen. The keys in turn were assigned, from top to bottom, to the index, middle, ring, and little finger of one hand and the index, middle, ring, and little finger of the other hand. The assignment of the left/right hand as the top or bottom hand was counterbalanced across participants.

During training, the imperative stimuli appearing in the tophand locations (T1, T2, T3, T4, from the top) followed a repeating sequence independent of that for stimuli in the bottom-hand locations (B4, B3, B2, B1, from the bottom), which appeared according to a different repeating sequence. We used an 8-element sequence (12313424) and a 9-element sequence (121341423), which together established a compound 144-element sequence (e.g., T1 B1 T2 B2 T3 B1 T1 B3 T3 B4 ...). This compound sequence is fully predictable only when 4 consecutive elements are taken into account: For each of the 32 possible combinations of 2 consecutive elements (2-tuples) there are at least 2 potential successor elements. The same is true for the sixty-eight 3-tuples, whereas there is only one possible successor for each 4-tuple.

When only one of the fixed, hand-related sequences was randomized, random trials for one hand alternated with sequence trials for the other hand. Each pseudorandom sequence was 72 elements long, so that it matched the number of trials through which the to-be-replaced hand-related sequence would have cycled over the length of an entire 144-trial test block. 
Furthermore, pseudorandom sequences were constructed to resemble the sequences they replaced, in that stimuli appeared with the same frequency and consecutive elements were never identical. From a large set of such pseudorandom sequences as many were selected as there were test blocks in the experiment, under the constraint that the selected pseudorandom sequences shared as few triples as possible with the to-be-replaced hand-related sequence. For example, out of the total of 70 triples (3-tuples) contained in each of those pseudorandom sequences selected to replace the 8 -element sequence, 12,15 , or 17 triples matched one of the 8 triples contained in the 8-element sequence. Similarly, each of those pseudorandom sequences replacing the 9-element sequence contained 17 triples (out of a total of 70) matching one of the 9 triples in the 9-element sequence.

Procedure. Participants were tested individually. Half of the participants practiced the 8 -element sequence with the left hand and the 9-element sequence with the right hand, and the assignment was reversed for the remaining participants. The experiment was conducted in three sessions scheduled for consecutive days. Session 1 started with a warm-up block in which pseudorandom sequences for both hands were presented, followed by 20 fixed-sequence blocks. Session 2 consisted of 21 fixed-sequence blocks. Session 3 comprised 15 blocks; the first 5 blocks were fixed-sequence blocks; beginning with the 6th block, 3 test blocks ( $L_{\text {rand }}, R_{\text {rand }}, L R_{\text {rand }}$ ) alternated with 3 fixed-sequence blocks. The order of presentation of these test blocks was counterbalanced across participants. Session 3 blocks 12 and 14 were test blocks of the $L_{\text {trans }}$ and $R_{\text {trans }}$ type. They were also embedded in fixedsequence blocks, and the order of their presentation was counterbalanced across participants independently of the counterbalancing of the previous 3 test blocks.

Each block comprised 144 trials (72 with the left hand and 72 with the right hand). Participants completed, on average, 414 repetitions of the 8 -element sequence and 368 repetitions of the 9-element sequence prior to encountering the first test block. Each fixed-sequence block began at a randomly determined position in the compound sequence. Each trial began with the presentation of the imperative stimulus. Following the participant's response, 140 ms elapsed before the next trial was initiated. In case of a response error, the German word for error (Fehler) appeared in red centered below the row of location squares. It was accompanied by a short beep tone for the duration of this response-stimulus interval.

Prior to each session, participants received written instructions, presented on the screen, informing them about the assignment of locations on the screen to keys on the keyboard and to fingers of the two hands, as described above. No mention was made of sequences. Both speed and accuracy were stressed in the instructions. Participants rested for at least $15 \mathrm{~s}$ between blocks and received feedback about their mean RT as well as the number of errors in the previous block.

\section{Results}

RTs from error trials $(4.2 \%)$ were excluded from analysis, as were RTs more than $3 S D$ above or below the mean RT as determined separately for each participant, each block, and each hand $(1.4 \%)$.

For each family of pairwise comparisons, $p$ values were subjected to the Bonferroni adjustment. Unless otherwise noted, all single-sample $t$ tests and all pairwise comparisons are one-tailed whenever directional hypotheses have been formulated. Whenever necessary, the degrees of freedom in repeated-measures analyses of variance (ANOVAs) were adjusted with the GreenhouseGeisser epsilon $\left(\varepsilon_{\mathrm{GG}}\right)$ in order to correct for any significant violations (Mauchly test) of the sphericity assumption. If such a correction has been carried out, the unadjusted degrees of freedom are reported together with the respective $\varepsilon_{\mathrm{GG}}$, and the reported $p$ value reflects the adjusted degrees of freedom.

Mean RTs and error rates in each block were computed separately for left-hand and for right-hand responses (see Table 2). RT costs as an index of sequence learning were computed as the difference between the mean RT in a test block and the mean of the mean RTs in the corresponding baseline blocks (Session 3 blocks $5,7,9$, and 11 for test blocks $L_{\text {rand }}, R_{\text {rand }}$, and $L R_{\text {rand }}$; Session 3 blocks 11,13 , and 15 for test blocks $L_{\text {trans }}$ and $R_{\text {trans }}$ ). Error costs were computed in an analogous manner. For relevant mean RT costs and mean error costs see Figure 2.

Randomization probes: $R T$ cost. There were significant lefthand RT costs when the left-hand sequence was randomized $\left(L_{\text {rand }}\right.$ and $\left.L R_{\text {rand }}\right)$, both $t \mathrm{~s}(23)>5.36$, both $p \mathrm{~s} \leq .001$, but also when the right-hand sequence was randomized $\left(R_{\text {rand }}\right), t(23)=2.45, p \leq$ .05 . There were significant right-hand RT costs only when the right-hand sequence was randomized ( $R_{\text {rand }}$ and $\left.L R_{\text {rand }}\right)$, both $t \mathrm{~s}(23)>7.11$, both $p \mathrm{~s} \leq .001$, but not when the left-hand sequence was randomized $\left(L_{\text {rand }}\right), t(23)=0.79$. This pattern of hand-related RT costs is reflected in a significant Hand $\times$ Test Block interaction, $F(2,46)=14.71, p \leq .001, \eta_{p}^{2}=.390$. The significant main effect of test block, $F(2,46)=5.41, p \leq .01, \eta_{p}^{2}=.190$, is an artifact of averaging across both hands in test blocks in which costs occurred for both hands versus test blocks in which costs occurred primarily for one hand but not the other. The main effect of hand was not significant, $F(1,23)=0.43, \eta_{p}^{2}=.018$.

Separate analyses were computed for left-hand and for righthand RT costs in order to unpack the significant interaction. These

Table 2

Mean RTs and Error Rates for Each Hand in the Different Baseline and Test Blocks in Experiment 1

\begin{tabular}{|c|c|c|c|c|}
\hline \multirow[b]{2}{*}{ Block } & \multicolumn{2}{|c|}{$\mathrm{RT}, \mathrm{ms}$} & \multicolumn{2}{|c|}{ Error rate, $\%$} \\
\hline & Left hand & Right hand & Left hand & Right hand \\
\hline \multicolumn{5}{|l|}{ Baseline for } \\
\hline $\begin{array}{l}\text { randomization } \\
\text { probes }\end{array}$ & $507.6(19.2)$ & $494.9(16.5)$ & $4.0(0.6)$ & $3.8(0.5)$ \\
\hline$L_{\text {rand }}$ & $530.0(18.4)$ & $498.7(16.2)$ & $5.4(0.9)$ & $3.9(0.6)$ \\
\hline$R_{\text {rand }}$ & $519.3(18.5)$ & $525.3(16.9)$ & $4.1(0.5)$ & $5.7(0.8)$ \\
\hline$L R_{\text {rand }}$ & $534.2(18.6)$ & $527.0(16.2)$ & $5.4(0.7)$ & $5.2(0.9)$ \\
\hline \multicolumn{5}{|l|}{ Baseline for transfer } \\
\hline probes & $497.4(19.4)$ & $483.4(16.1)$ & $3.8(0.5)$ & $3.9(0.5)$ \\
\hline$L_{\text {trans }}$ & $517.2(17.9)$ & $500.7(16.9)$ & $4.7(0.7)$ & $4.3(0.8)$ \\
\hline$R_{\text {trans }}$ & $518.1(19.8)$ & $514.8(16.6)$ & $5.2(0.7)$ & $6.8(0.8)$ \\
\hline
\end{tabular}

Note. Standard errors are shown in parentheses. $\mathrm{RT}=$ reaction time; $L_{\mathrm{rand}}=$ left-hand sequence randomized, right-hand sequence retained; $R_{\text {rand }}=$ righthand sequence randomized, left-hand sequence retained; $L R_{\text {rand }}=$ both hand-related sequences randomized; $L_{\text {trans }}=$ left-hand sequence transferred to the right hand, randomized sequence for the left hand; $R_{\text {trans }}=$ righthand sequence transferred to the left hand, randomized sequence for the right hand. 

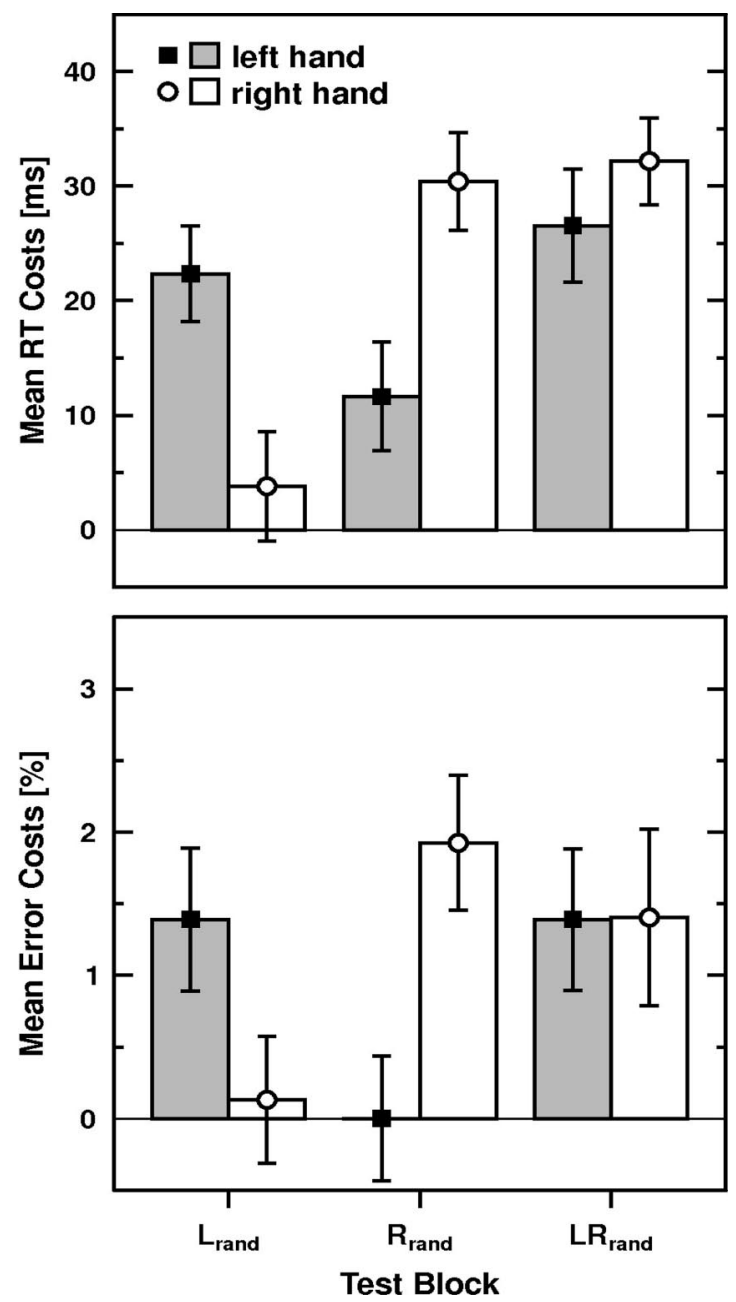

Figure 2. Mean reaction time (RT) costs and mean error costs $( \pm 1$ SEM, respectively) separately for the left and the right hand in test blocks in which the left-hand sequence, the right-hand sequence, or both handrelated sequences were randomized ( $L_{\text {rand }}, R_{\text {rand }}, L R_{\text {rand }}$, respectively) in Experiment 1.

analyses revealed significant main effects of test block both for left-hand and for right-hand RT costs, both $F \mathrm{~s}(2,46)>3.69$, both $p \mathrm{~s} \leq .05$, both $\eta_{p}^{2}>.138$. Pairwise comparisons revealed that left-hand RT costs did not differ significantly between test blocks $L_{\text {rand }}$ and $L R_{\text {rand }}, t(23)=0.81$, or between test blocks $L_{\text {rand }}$ and $R_{\text {rand }}, t(23)=1.75, p \leq .14$, but they were significantly higher in test block $L R_{\text {rand }}$ than in test block $R_{\text {rand }}, t(23)=2.66, p \leq .01$. Right-hand RT costs did not differ between test blocks $R_{\text {rand }}$ and $L R_{\text {rand }}, t(23)=0.31$, but they were significantly higher in these test blocks than in test block $L_{\text {rand }}$, both $t \mathrm{~s}(23)>3.74$, both $p \mathrm{~s} \leq$ .005 .

Randomization probes: Error costs. There were significant left-hand error costs when the left-hand sequence was randomized $\left(L_{\text {rand }}\right.$ and $\left.L R_{\text {rand }}\right)$, both $t \mathrm{~s}(23)>2.78$, both $p \mathrm{~s} \leq .01$, but not when the right-hand sequence was randomized $\left(R_{\text {rand }}\right), t(23)<0.01$. There were significant right-hand error costs when the right-hand sequence was randomized $\left(R_{\text {rand }}\right.$ and $\left.L R_{\text {rand }}\right)$, both $t \mathrm{~s}(23)>2.27$, both $p \mathrm{~s} \leq .05$, but not when the left-hand sequence was random- ized $\left(L_{\text {rand }}\right), t(23)=0.29$. This pattern of hand-related RT costs is reflected in a significant Hand $\times$ Test Block interaction, $F(2$, 46) $=4.86, p \leq .05, \eta_{p}^{2}=.174$. Neither the main effect of hand, $F(1,23)=0.17$, nor the main effect of test block, $F(2,46)=1.34$, was significant, both $\eta_{p}^{2}<.055$.

Separate analyses were computed for left-hand and for righthand error costs in order to unpack the significant interaction. These analyses revealed significant main effects of test block both for left-hand and for right-hand error costs, both $F_{\mathrm{s}}(2,46)>3.19$, both $p$ s $\leq .05$, both $\eta_{p}^{2}>.121$. Pairwise comparisons revealed that left-hand error costs did not differ significantly between test blocks $L_{\text {rand }}$ and $L R_{\text {rand }}, t(23)<0.01$, or between test blocks $R_{\text {rand }}$ and $L R_{\text {rand }}, t(23)=2.06, p \leq .076$, but they were significantly higher in test block $L_{\text {rand }}$ than in test block $R_{\text {rand }}, t(23)=2.26, p \leq .05$. Right-hand error costs differed neither between test blocks $R_{\text {rand }}$ and $L R_{\text {rand }}, t(23)=0.91$, nor between test blocks $L_{\text {rand }}$ and $L R_{\text {rand }}$, $t(23)=1.64$, but they were significantly higher in test block $R_{\text {rand }}$ than in test block $L_{\text {rand }}, t(23)=2.85, p \leq .05$.

Transfer probes: $R T$ costs. Significant left-hand and righthand RT costs were observed in both transfer blocks $L_{\text {trans }}$ and $R_{\text {trans }}$, all $t \mathrm{~s}(23)>3.45$, all $p \mathrm{~s} \leq .005$. A Hand $\times$ Test Block repeated-measures ANOVA revealed a significant main effect of test block, $F(2,46)=3.93, p \leq .05, \eta_{p}^{2}=.146$, indicating that RT costs were higher in test block $L R_{\text {rand }}$ than in transfer block $L_{\text {trans }}$, $t(23)=3.47, p \leq .005$, but not significantly higher than in transfer block $R_{\text {trans }}, t(23)=0.84$; there was no significant difference between RT costs in transfer blocks $L_{\text {trans }}$ and $R_{\text {trans }}$ either, $t(23)=$ 1.61. There was no significant Hand $\times$ Test Block interaction, $F(2$, 46) $=1.39, \eta_{p}^{2}=.057$ (i.e., no indication of a pattern of RT costs specific to one hand but not the other). The main effect of hand was not significant either, $F(1,23)=1.69, \eta_{p}^{2}=.068$.

Transfer probes: Error costs. Transfer block error costs were significant for both hands in transfer block $R_{\text {trans }}$, both $t \mathrm{~s}(23)>$ 2.58 , both $p \mathrm{~s} \leq .05$, but not in transfer block $L_{\text {trans }}$, both $t \mathrm{~s}(23)<$ 1.67 , both $p s>.054$. An analysis of variance analogous to that on RT costs revealed a significant main effect of test block, $F(2$, 46) $=5.07, p \leq .01, \eta_{p}^{2}=.181$, indicating that error costs were higher in transfer block $R_{\text {trans }}$ than in test block $L_{\text {trans }}, t(23)=3.71$, $p \leq .005$, whereas error costs differed neither between blocks $L R_{\text {rand }}$ and $L_{\text {trans }}$ nor between blocks $L R_{\text {rand }}$ and $R_{\text {trans, }}$, both $t \mathrm{~s}(23)<1.56$. The Hand $\times$ Test Block interaction missed significance, $F(2,46)=2.51, p \leq .093, \eta_{p}^{2}=.098$. The main effect of hand was not significant either, $F(1,23)=0.51, \eta_{p}^{2}=.022$.

\section{Discussion}

Participants performed an SRT task in which actions of both hands alternated and each hand performed its own fixed sequence of actions. After extensive practice, when we randomized one of the two hand-related sequences and retained the other, performance deteriorated primarily for that hand that lost its sequence, and performance with the other hand was largely unaffected. This pattern of RT and error costs, which is consistent with independent learning of the hand-related sequences, emerged most clearly for the right hand and in somewhat diluted form for the left hand. The emergence of left-hand RT costs upon randomization of the righthand sequence (i.e., in test block $R_{\text {rand }}$ ) might be taken to reflect integrated learning of the compound sequence. It is not obvious, however, why integrated learning should be evident only for the 
left hand in test block $R_{\text {rand }}$ but not for the right hand in test block $L_{\text {rand }}$. Possibly, learning with the dominant hand (i.e., the right hand in this experiment's sample of mostly right-dominant participants) occurs more independently from learning with the nondominant (left) hand than vice versa. Alternatively, changes in the sequence performed with the dominant (i.e., right) hand might be more salient to participants than are changes in the sequence performed with the nondominant (i.e., left) hand, so that upon randomization of the right-hand sequence participants would adopt a more cautious response set; this in turn would result in left-hand RT costs. In contrast, randomization of the left-hand sequence might not be as salient to participants and consequently would not cause them to adopt a more cautious response set; thus, it would not result in right-hand $\mathrm{RT}$ costs. ${ }^{2} \mathrm{Be}$ that as it may, the overall data pattern suggests that the two hand-related sequences were learned at least partly independently of each other.

Because the hand-related sequences used in Experiment 1 were uncorrelated, elements from one hand were not predictive of subsequent elements for the other hand (across-hands transitions) above and beyond the predictive value already afforded by previous elements from the same hand (within-hand transitions). In a sense, then, independent learning of hand-related sequences is tantamount to the concurrent learning of hand-related gap-2 conditionals (i.e., contingencies in which the identity of element $n$ depends on the identity of element $n-2$, regardless of the identity of element $n$-1). Learning of such gap-2 conditionals has been investigated by Howard and colleagues (J. H. Howard \& Howard, 1997; see also D. V. Howard et al., 2004; J. H. Howard, Howard, Dennis, Yankovich, \& Vaidya, 2004; Japikse et al., 2003): In what they termed an alternating SRT (ASRT) task, sequence trials alternated with random trials so that every other stimulus and, thus, every other response was part of a repeating sequence. The ASRT task further differs from the setup used here inasmuch as sequence stimuli appeared in the same locations as random stimuli and participants used the same fingers on the same keys for responding both to sequence stimuli and to random stimuli.

The vertical arrangement of stimulus locations and response keys allowed us to arrange intermanual transfer in such a way that the practiced sequence was conserved not only in terms of visualspatial coordinates but also in terms of homologous fingers. Even so, there was no clear advantage of executing the transferred sequence with the hand with which the other sequence had been practiced relative to executing a random sequence. Numerically, the pattern of right-hand error costs in particular suggests that performance with the right hand was not disrupted much when responding to the left-hand sequence in transfer block $L_{\text {trans }}$, whereas performance appears to have clearly suffered when responding to a random sequence in transfer block $R_{\text {trans }}$. However, no corresponding pattern emerged for the left hand, and the critical Hand $\times$ Test Block interaction observed for randomization blocks was not significant. This lack of evidence for intermanual transfer is consistent with the view that any independently acquired sequence knowledge is represented in a hand-specific manner. This conclusion has to be drawn with some caution, however. Although significant intermanual transfer has been obtained under very similar conditions in a bimanual-bisequential SRT task (Berner \& Hoffmann, 2008), the conditions employed here might not have been optimal for intermanual transfer. In particular, the possibility exists that concurrently responding to a random sequence with the hand with which the to-be-transferred sequence has been practiced might interfere with intermanual transfer.

Prompted by concerns voiced by an anonymous reviewer about whether the pattern of results obtained in Experiment 1 might also have resulted from purely integrated learning of the compound sequence, we have analyzed in detail the way in which randomizing only one of the hand-related sequences while retaining the other affects the compound sequence. For each block, we divided the compound sequence (e.g., 34251426 ...) into all possible 2-tupels (e.g., 34, 42, 25, 51), 3-tupels (e.g., 342, 425, 251, 514), 4-tupels (e.g., 3425, 4251, 2514, 5142), and 5-tupels (e.g., 34251, $42514,25142,51426)$. Then, we determined for each test and transfer block the number of deviant tupels that did not occur in regular baseline blocks. Those tupels ending with (i.e., transitioning to or "predicting") a right-hand response (e.g., 34, 25, 425, $514,3425,2514,42514,51426)$ were counted separately from those tupels ending with a left-hand response (e.g., 42, 51, 342, 251, 4251, 5142, 34251, 25142). For odd-element tupels from the compound sequence (i.e., 3-tupels, 5-tupels; generally, tupels whose first and last elements pertain to the same hand-related sequence/hand), randomization primarily altered transitions to stimuli/responses pertaining to the randomized sequence, whereas transitions to stimuli/responses pertaining to the maintained sequence were comparatively spared. Table 3 provides an overview.

For example, in test block $L_{\text {rand }}$ the number of deviant oddelement tupels is higher for tupels transitioning to a sequence element pertaining to the left hand than for tupels transitioning to a sequence element pertaining to the right hand. The reverse is true for test block $R_{\text {rand }}$. In other words, randomizing only one of the hand-related sequences altered particularly those transitions in the compound sequence that pertain to the hand losing its sequence, whereas those transitions in the compound sequence that pertain to the other hand were relatively spared. Although no such differences exist for even-element tupels (i.e., 2-tupels, 4-tupels) in any of the test blocks, the pattern of hand-specific randomization costs as observed in Experiment 1 could have emerged from purely integrated learning of the compound sequence, because the higher the number of deviant transitions, the more performance is going to be disrupted (i.e., higher RT/error costs).

An account assuming purely integrated learning of the compound sequence is also consistent with the fact that there were no indications for intermanual transfer. However, unless participants' learning of the compound sequence was restricted to first- and second-order transitions-which seems somewhat unlikely, given that it has been shown that people can learn up to fourth-order sequential dependencies (Remillard, 2008; see also Remillard \&

\footnotetext{
${ }^{2}$ We thank an anonymous reviewer for suggesting this interpretation, which can be linked to work by Jiménez, Vaquero, and Lupiáñez (2006). These authors demonstrated that introducing a decrease in the proportion of sequence transitions adhering to a previously practiced sequential regularity selectively hindered the expression of intentionally (vs. incidentally) acquired sequence knowledge on trials for which the sequential regularity was maintained. Although our Experiment 1 did not feature a manipulation of intentional versus incidental learning instructions, it can be speculated in analogy to Jiménez et al. (2006) that the disruptive effects of randomizing a previously practiced sequence are more pronounced for the possibly more salient sequential regularity pertaining to the dominant (i.e., right) hand than for the nondominant (i.e., left) hand.
} 
Table 3

Mean Number of Deviant 2-, 3-, 4-, and 5-Tupels in the Various Test and Transfer Blocks in Experiment 1 Separately for the Left Hand and the Right Hand

\begin{tabular}{|c|c|c|c|c|c|c|c|c|}
\hline \multirow[b]{2}{*}{ Block } & \multicolumn{2}{|c|}{ 2-tupel } & \multicolumn{2}{|c|}{ 3-tupel } & \multicolumn{2}{|c|}{ 4-tupel } & \multicolumn{2}{|c|}{ 5-tupel } \\
\hline & Left hand & Right hand & Left hand & Right hand & Left hand & Right hand & Left hand & Right hand \\
\hline$L_{\text {rand }}$ & $00.0(0.0)$ & $00.0(0.0)$ & $21.0(1.5)$ & $00.0(0.0)$ & $20.8(1.4)$ & $20.5(1.4)$ & $53.0(0.0)$ & $20.3(1.4)$ \\
\hline$R_{\text {rand }}$ & $00.0(0.0)$ & $00.0(0.0)$ & $00.0(0.0)$ & $21.0(1.5)$ & $20.6(1.5)$ & 20.7 (1.4) & $20.3(1.4)$ & $53.0(0.0)$ \\
\hline$L R_{\text {rand }}$ & $00.0(0.0)$ & $00.0(0.0)$ & $19.0(1.0)$ & $19.0(1.0)$ & $32.3(0.4)$ & $32.4(0.4)$ & $57.0(0.2)$ & $57.0(0.1)$ \\
\hline$L_{\text {trans }}$ & $00.0(0.0)$ & $00.0(0.0)$ & $20.5(0.9)$ & $12.5(0.7)$ & $29.3(0.5)$ & $29.5(0.3)$ & $58.2(0.3)$ & $59.0(0.3)$ \\
\hline$R_{\text {trans }}$ & $00.0(0.0)$ & $00.0(0.0)$ & $12.5(0.7)$ & $20.5(0.9)$ & $29.3(0.3)$ & $29.3(0.5)$ & $58.8(0.3)$ & $58.1(0.3)$ \\
\hline
\end{tabular}

Note. Standard errors are shown in parentheses. $L_{\text {rand }}=$ left-hand sequence randomized, right-hand sequence retained; $R_{\text {rand }}=$ right-hand sequence randomized, left-hand sequence retained; $L R_{\text {rand }}=$ both hand-related sequences randomized; $L_{\text {trans }}=$ left-hand sequence transferred to the right hand, randomized sequence for the left hand; $R_{\text {trans }}=$ right-hand sequence transferred to the left hand, randomized sequence for the right hand.

Clark, 2001)—integrated learning should have engendered righthand performance costs upon randomization of the left-hand sequence, which was not the case. Yet, integrated learning of the compound sequence should have been very difficult and might have been only partial due to the length and complexity of the compound sequence implemented in Experiment 1; this in turn might have favored independent learning of the constituent handrelated sequences.

In Experiment 2 we set out to investigate independent learning of hand-related sequences under conditions that make integrated learning more likely by combining hand-related sequences of the same length so that across-hands transitions actually possess predictive value above and beyond within-hand transitions. Additionally, these conditions make available another test of independent learning that does not rely on sequence randomization and thus avoids the problem of hand-specific increases in the number of deviant compound-sequence transitions upon hand-specific randomization. Nevertheless, we eliminated this problem in randomization test blocks by constructing the random sequences accordingly. Finally, to improve conditions for intermanual transfer, we avoided possible interference from alternating bimanual responding by testing for intermanual transfer under conditions of unimanual responding.

\section{Experiment 2}

The major change made in Experiment 2 compared to Experiment 1 was that we chose two hand-related sequences that are equal in length. This allows for a new kind of test for independent learning: When the hand-related sequences are shifted relative to each other (cf. Schmidtke \& Heuer, 1997), between-hand regularities are destroyed and within-hand regularities remain intact. As a consequence, performance in such a shift probe should not suffer at all if the two hand-related sequences are learned entirely independently of one another. In contrast, to the extent that integrated learning of the compound sequence occurs, which pertains to between-hand regularities, performance should deteriorate in such a shift probe.

The correlated constituent sequences together establish a compound sequence that - although still twice as long-is similar to its constituent sequences in terms of statistical structure. Integrated learning was more likely to occur than in Experiment 1 because the compound sequence in Experiment 2 was much shorter and con- siderably less complex than the compound sequence in Experiment 1. The conditions for integrated learning were further put on par with the conditions for independent learning in this experiment compared with Experiment 1, insofar as the use of correlated hand-related sequences eliminated the discrepancy in terms of the extent of practice between the hand-related sequences and the compound sequence; as a result of the hand-related sequences being intertwined, every single repetition of each of the handrelated sequences was tantamount to a single repetition of the compound sequence. Test blocks were inserted in Session 2 as well as at the end of Session 3 in order to explore the time course of acquisition of integrated versus independent learning.

\section{Method}

Experiment 2 was very similar to Experiment 1 in several respects. Only the pertinent differences are described.

Participants. A total of 24 individuals (mean age $=23$ years) volunteered to participate in Experiment 2 to partially fulfill course requirements or for payment of 20 euros. Of the participants, 20 reported they were predominantly right-handed, and 4 professed they were ambidextrous.

Task and design. The task in the main part of Experiment 2 was the same as that in Experiment 1. The same types of test blocks were implemented as in Experiment 1, except that there were no transfer blocks. We introduced an additional test block that was also suited to investigating independent versus integrated sequence learning. In this test block, the two hand-related sequences were shifted against each other $\left(L R_{\text {shift }}\right.$; cf. Schmidtke \& Heuer, 1997), so that the position of one sequence (e.g., _W_X_Y_Z ...) relative to the other sequence (e.g., A_B_C_D_...) established a compound sequence different from the one present in regular blocks (e.g., AYBZCWDX ... instead of AWBXCYDZ ...). At the end of the experiment, participants engaged in a unimanual SRT task involving vertically aligned stimulus locations and keys. The hand-related sequences practiced during the bimanual phase of the experiment were mapped to the locations/keys in such a way that intermanual transfer pertained to homologous fingers.

Apparatus and materials. The bimanual phase was essentially identical to Experiment 1 except that the keys were horizontally aligned, as were locations on the screen. Each location was marked by a line (14 $\mathrm{mm}$ wide), and there was an equal distance $(6 \mathrm{~mm})$ 
between any two adjacent lines. The keys were assigned to the lines (locations) from left to right. The keys in turn were assigned from left to right to the little, ring, middle, and index finger of the left hand and the index, middle, ring, and little finger of the right hand.

During the bimanual training phase, the imperative stimuli appearing in the left-hand-side locations (L1, L2, L3, L4, from left to right) followed a repeating sequence independent of that for stimuli in the right-hand-side locations (R1, R2, R3, R4, from left to right), which appeared according to a different repeating sequence. We used two 8-element sequences (Sequence A, 14232134; Sequence B, 31342124), which together established a compound 16-element sequence (AB = L1 R3 L4 R1 L2 R3 L3 R4 L2 R2 L1 R1 L3 R2 L4 R4). Although the compound sequence (a) extends across twice as many positions and (b) is twice as long as its constituent sequences, the 16-element sequence and the 8-element sequences are quite similar in terms of statistical structure. Like Sequences $\mathrm{A}$ and $\mathrm{B}$, Sequence $\mathrm{AB}$ contains no unique transitions (i.e., for each element in the sequence there are two possible successor elements), so that the next element can be predicted only from the current and the previous element (i.e., second-order transitions).

We constructed a large set of pseudorandom sequences according to the same constraints described for Experiment 1, taking into account that each block comprised 128 trials (64 with the left hand and 64 with the right hand). From this set we selected pseudorandom sequences so that randomization created the same number of deviant compound-sequence transitions for both hands (see Table 4). Out of the total of 62 triples contained in each of the pseudorandom sequences selected to replace Sequence A, 8, 9, 12, or 17 triples matched one of the 8 triples contained in Sequence A. Similarly, each of those pseudorandom sequences replacing Sequence B contained $9,14,15$, or 16 triples (out of a total of 62) that matched one of the 8 triples in Sequence B. No pseudorandom sequences were needed for $L R_{\text {shift }}$ test blocks in which, compared to no-shift blocks, Sequence B (31342124) was shifted four elements (i.e., 21243134) relative to Sequence A (14232134).

In the unimanual task, participants responded on four vertically aligned keys that were connected to the computer via the parallel port. Accordingly, there were four vertically aligned locations (1, $2,3,4$, from top to bottom) on the screen, and each was marked by a square outlined in black (side length $=22 \mathrm{~mm}$ ). There was a distance of $6 \mathrm{~mm}$ between any two adjacent squares. Keys were assigned compatibly to the squares. The keys were assigned from top to bottom to the index, middle, ring, and little finger of either hand. The imperative stimulus consisted of the inside area of the square turning turquoise. The changes in stimulus presentation and the vertical arrangement of keys served to minimize overlap with the bimanual phase of the experiment in terms of visual-spatial coordinates of sequence representations. The hand-related structured sequences practiced during the bimanual phase were presented in the locations in such a way that the sequence of fingers to be used was preserved. Thus, as in Experiment 1, the vertical arrangement allows for intermanual transfer between homologous fingers. The structured sequences were embedded in 12-trial pseudorandom sequences. These pseudorandom sequences were constructed under the same constraints as those in the bimanual phase of the experiment, and they established the baseline against which structured-sequence performance was to be compared.

Procedure. The assignment of Sequences A and B to the left and the right hand was counterbalanced across participants. The bimanual phase of the experiment was conducted in three sessions, each of which comprised 22 blocks. The sessions were scheduled for consecutive days. Session 1 started with a warm-up block in which pseudorandom sequences were presented for both hands, followed by 21 fixed-sequence blocks. Except for the test blocks described below, all Session 2 and Session 3 blocks were fixedsequence blocks. Beginning with the 4th block in Session 2 and with the 11th block in Session 3, four test blocks ( $L_{\text {rand }}, R_{\text {rand }}$, $\left.L R_{\text {rand }}, L R_{\text {shift }}\right)$ alternated with four fixed-sequence blocks. The order of presentation of these test blocks was counterbalanced across participants, with the additional constraint that none of the participants saw the same order of test blocks in Sessions 2 and 3.

Each block comprised 128 trials (64 with the left hand and 64 with the right hand), so that prior to Session 2 and Session 3 test blocks participants completed 192 and 392 repetitions of Sequences $\mathrm{A}$ and $\mathrm{B}$ as well as of the compound sequence, $\mathrm{AB}$. The number of sequence repetitions given above does not include

Table 4

Mean Number of Deviant 2-, 3-, 4-, and 5-Tupels in the Various Test Blocks in Sessions 2 and 3 of Experiment 2 Separately for the Left Hand and the Right Hand

\begin{tabular}{|c|c|c|c|c|c|c|c|c|}
\hline \multirow[b]{2}{*}{ Block } & \multicolumn{2}{|c|}{ 2-tupel } & \multicolumn{2}{|c|}{ 3-tupel } & \multicolumn{2}{|c|}{ 4-tupel } & \multicolumn{2}{|c|}{ 5-tupel } \\
\hline & Left hand & Right hand & Left hand & Right hand & Left hand & Right hand & Left hand & Right hand \\
\hline \multicolumn{9}{|l|}{ Session 2} \\
\hline$L_{\text {rand }}$ & $35.5(0.0)$ & $35.5(0.0)$ & $52.0(0.0)$ & $52.0(0.0)$ & $58.5(0.0)$ & $58.5(0.0)$ & $59.5(0.3)$ & $59.5(0.3)$ \\
\hline$R_{\text {rand }}$ & $35.5(0.0)$ & $35.5(0.0)$ & $52.0(0.0)$ & $52.0(0.0)$ & $58.5(0.0)$ & $58.5(0.0)$ & $60.0(0.4)$ & $60.0(0.4)$ \\
\hline$L R_{\text {rand }}$ & $34.6(0.1)$ & $34.8(0.1)$ & $52.0(0.0)$ & $52.0(0.0)$ & $58.5(0.0)$ & $58.5(0.0)$ & $61.0(0.0)$ & $61.0(0.0)$ \\
\hline$L R_{\text {shift }}$ & $43.5(0.9)$ & $43.5(0.9)$ & $55.0(1.8)$ & $55.0(1.8)$ & $62.5(0.0)$ & $62.5(0.0)$ & $62.0(0.0)$ & $62.0(0.0)$ \\
\hline \multicolumn{9}{|l|}{ Session 3} \\
\hline$L_{\text {rand }}$ & $35.5(0.0)$ & $35.5(0.0)$ & $52.0(0.0)$ & $52.0(0.0)$ & $58.5(0.0)$ & $58.5(0.0)$ & $59.0(0.2)$ & $59.0(0.2)$ \\
\hline$R_{\text {rand }}$ & $35.0(0.1)$ & $35.0(0.1)$ & $52.0(0.0)$ & $52.0(0.0)$ & $58.5(0.0)$ & $58.5(0.0)$ & $59.5(0.3)$ & $59.5(0.3)$ \\
\hline$L R_{\text {rand }}$ & $35.2(0.1)$ & $35.2(0.1)$ & $52.0(0.0)$ & $52.0(0.0)$ & $58.5(0.0)$ & $58.5(0.0)$ & $60.5(0.1)$ & $60.5(0.1)$ \\
\hline$L R_{\text {shift }}$ & $43.5(0.9)$ & $43.5(0.9)$ & $55.0(1.8)$ & $55.0(1.8)$ & $62.5(0.0)$ & $62.5(0.0)$ & $62.0(0.0)$ & $62.0(0.0)$ \\
\hline
\end{tabular}

Note. Standard errors are shown in parentheses. $L_{\text {rand }}=$ left-hand sequence randomized, right-hand sequence retained; $R_{\text {rand }}=$ right-hand sequence randomized, left-hand sequence retained; $L R_{\text {rand }}=$ both hand-related sequences randomized; $L R_{\text {shift }}=$ the two hand-related sequences shifted relative to each other. 
sequence repetitions in Session 2 test blocks $L_{\text {rand }}, R_{\text {rand, }}$ and $L R_{\text {shift }}$. Each fixed-sequence block began at a randomly selected position in the compound 16-element sequence established by the two hand-related sequences. The response-stimulus interval was $120 \mathrm{~ms}$.

The unimanual phase of the experiment commenced at the end of Session 3. Participants completed eight blocks. Each block comprised 50 trials. The first 12 trials and the last 12 trials of each block were pseudorandom. The trials in between consisted of three repetitions of a structured sequence, which were preceded by two additional structured trials for a total of 26 structured trials. The response-stimulus interval was $140 \mathrm{~ms}$. Before each block, participants received written instructions on the monitor about which hand to use in the upcoming block. Participants alternated between using the left and the right hand. Which hand participants started with was counterbalanced orthogonally to the assignment of sequences to the hands in the bimanual phase of the experiment. The structured sequence presented to participants was either the sequence they had previously (i.e., in the bimanual phase) practiced with the same hand (standard probe) or the sequence they had previously practiced with the other hand (transfer probe). For each hand there were two standard probe blocks and two transfer probe blocks. For each hand the order of these blocks was counterbalanced across participants according to a Latin square scheme, so that the Latin squares for the two hands formed a Graeco-Latin square.

\section{Results}

Analyses of data from the bimanual phase of the experiment are presented first and are followed by results from the intermanual transfer test in the unimanual phase.

Bimanual phase. RTs from error trials (4.3\%) as well as outlier RTs (1.5\%) were excluded from analysis. Mean RTs and mean error frequencies are given in Table 5. RT costs and error costs in randomization blocks were computed as described for Experiment 1. RT costs in shift probes were computed relative to the same baseline blocks used for randomization costs. Analyses of Experiment 2 error data, unlike those for Experiment 1, did not provide additional insights over and above what is evident in analyses of RT data. Also, there were no indications of a speedaccuracy trade-off. Therefore, error data and their analyses are not reported here in detail. Analyses of data from randomization probes are presented first; then, analyses of data from the newly introduced shift probes are reported.

Randomization probes. There were significant left-hand and right-hand RT costs in all test blocks ( $L_{\text {rand }}, R_{\text {rand }}$, and $\left.L R_{\text {rand }}\right)$ in both sessions, all $t \mathrm{~s}(23)>11.27$, all $p \mathrm{~s} \leq .001$. An ANOVA with repeated measures on the factors session $(2,3)$, hand (left, right), and test block ( $\left.L_{\text {rand }}, R_{\text {rand }}, L R_{\text {rand }}\right)$ revealed a significant Hand $\times$ Test Block interaction, $F(2,46)=21.86, p \leq .001, \eta_{p}^{2}=.487$. The significant main effect of session, $F(1,23)=115.68, p \leq .001$, $\eta_{p}^{2}=.834$, reflects that, overall, RT costs were higher in Session $3(M=220.3 \mathrm{~ms}, S E=10.8)$ than in Session $2(M=146.4 \mathrm{~ms}$, $S E=9.8)$. However, the Hand $\times$ Test Block interaction was not modulated by session, $F(2,46)=0.36, \eta_{p}^{2}=.015$. The Session $\times$ Test Block interaction approached significance, $F(2,46)=2.85$, $p \leq .068, \eta_{p}^{2}=.110$. No other main effects or interactions were significant, all $F \mathrm{~s}<0.56$, all $p \mathrm{~s}>.158$, all $\eta_{p}^{2}<.129$.
Table 5

Mean RTs and Error Rates for Each Hand in the Baseline and Test Blocks in Sessions 2 and 3 of Experiment 2

\begin{tabular}{cccccc}
\hline & \multicolumn{2}{c}{ RT, ms } & & \multicolumn{2}{c}{ Error rate, \% } \\
\cline { 2 - 3 } Block & Left hand & Right hand & & Left hand & Right hand \\
\hline Session 2 & & & & \\
Baseline & & & & \\
blocks & $397.9(18.5)$ & $394.0(16.3)$ & & $3.1(0.4)$ & $3.2(0.3)$ \\
$L_{\text {rand }}$ & $550.9(13.4)$ & $525.5(13.8)$ & & $7.6(0.7)$ & $6.8(0.9)$ \\
$R_{\text {rand }}$ & $535.4(13.6)$ & $542.3(14.8)$ & & $7.0(0.9)$ & $6.7(0.9)$ \\
$L R_{\text {rand }}$ & $552.1(14.6)$ & $547.9(14.5)$ & $7.4(0.9)$ & $6.4(0.9)$ \\
$L R_{\text {shift }}$ & $518.4(12.2)$ & $523.7(15.7)$ & $6.4(1.0)$ & $5.8(0.9)$ \\
Session 3 & & & & \\
Baseline & & & & \\
blocks & $292.2(18.9)$ & $291.5(18.1)$ & & $2.8(0.4)$ & $3.9(0.6)$ \\
$L_{\text {rand }}$ & $525.0(13.8)$ & $501.3(15.4)$ & & $9.2(1.0)$ & $9.1(1.1)$ \\
$R_{\text {rand }}$ & $512.0(17.3)$ & $515.9(14.6)$ & $7.7(0.8)$ & $9.0(1.1)$ \\
$L R_{\text {rand }}$ & $507.6(14.5)$ & $511.1(15.8)$ & & $8.4(1.0)$ & $7.7(0.9)$ \\
$L R_{\text {shift }}$ & $489.0(13.9)$ & $486.2(14.6)$ & $7.9(1.0)$ & $7.2(1.0)$ \\
\hline
\end{tabular}

Note. Standard errors are shown in parentheses. $\mathrm{RT}=$ reaction time; $L_{\text {rand }}=$ left-hand sequence randomized, right-hand sequence retained; $R_{\text {rand }}=$ righthand sequence randomized, left-hand sequence retained; $L R_{\text {rand }}=$ both hand-related sequences randomized; $L R_{\text {shift }}=$ the two hand-related sequences shifted relative to other.

Separate analyses were computed for left-hand and for righthand RT costs in order to unpack the significant Hand $\times$ Test Block interaction. These analyses revealed significant main effects of test block both for left-hand RT costs, $F(2,46)=4.75, p \leq .05$, $\eta_{p}^{2}=.171$, and for right-hand RT costs, $F(2,46)=7.44, p \leq .005$, $\eta_{p}^{2}=.245$. Left-hand RT costs did not differ significantly between test blocks $L_{\text {rand }}$ and $L R$ rand $t(23)=1.69$, nor between test blocks $R_{\text {rand }}$ and $L R_{\text {rand }}, t(23)=1.43$, but were significantly higher in test block $L_{\text {rand }}$ than in test block $R_{\text {rand }}, t(23)=2.97, p \leq .05$. Right-hand RT costs did not differ significantly between test blocks $R_{\text {rand }}$ and $L R_{\text {rand }}, t(23)=0.09$, but were significantly higher in each of these test blocks than in test block $L_{\text {rand }}$, both $t \mathrm{~s}(23)>$ 3.12 , both $p s \leq .05$. In other words, performance with the left/right hand generally deteriorated more pronouncedly when the left/right hand lost its sequence than when it retained its sequence while the right/left hand lost its sequence (see Figure 3).

Shift probes. Shifting the hand-related sequences produced costs in both sessions, all $t \mathrm{~s}(23)>9.51$, all $p \mathrm{~s} \leq .001$. As can be seen in Figure 4, shift costs were smaller than randomization costs in block $L R_{\text {rand }}, F(1,23)=35.60, p \leq .001, \eta_{p}^{2}=.608$. This shift block advantage did not vary significantly between sessions, $F(1$, $23)=0.53, \eta_{p}^{2}=.022$, but was significant in Session $2, F(1,23)=$ 14.77, $p \leq .001, \eta_{p}^{2}=.391$, as well as in Session 3, $F(1,23)=$ 16.56, $p \leq .001, \eta_{p}^{2}=.419$. Also, there were no differences between the two hands in terms of the shift block advantage, all $F \mathrm{~s}(1,23)<1.58$, all $\eta_{p}^{2}<.065$.

Unimanual phase: Transfer probes. RTs from error trials $(4.2 \%)$ as well as outlier RTs $(1.4 \%)$ were excluded from analysis. Also, we discarded the first two trials of each 26-trial structured run, because for both structured sequences each element is fully determined only by the preceding two elements in the sequence. The remaining RTs were averaged separately for random trials and structured trials. 


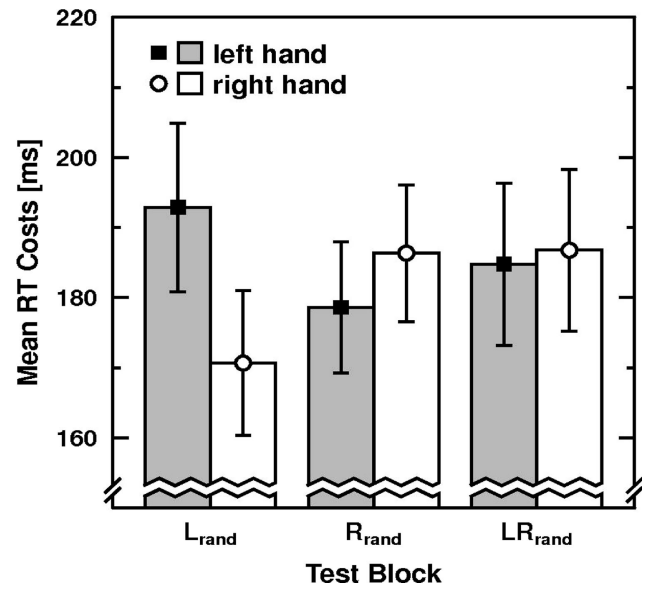

Figure 3. Mean reaction time (RT) costs ( \pm 1 SEM, respectively) separately for the left and the right hand in test blocks in which the left-hand sequence, the right-hand sequence, or both hand-related sequences were randomized ( $L_{\text {rand }}, R_{\text {rand }}, L R_{\text {rand }}$, respectively) in Experiment 2.

Mean RTs were analyzed in a 2 (Hand: left, right) $\times 2$ (Probe: standard, transfer) $\times 2$ (Sequence: structured, random) repeatedmeasures ANOVA. This analysis yielded a significant main effect of sequence, $F(1,23)=21.47, p \leq .001, \eta_{p}^{2}=.483$, that reflected faster responses on structured trials $(M=448.5 \mathrm{~ms}, S E=14.0)$ than on random trials $(M=464.3 \mathrm{~ms}, S E=14.2)$. Furthermore, the Probe $\times$ Sequence interaction was significant, $F(1,23)=$ $15.05, p \leq .001, \eta_{p}^{2}=.396$. No other main effects or interactions were significant, all $F_{\mathrm{s}}(1,23)<2.19$, all $p \mathrm{~s}>.152$, all $\eta_{p}^{2}<.087$. The significant Probe $\times$ Sequence interaction (see Figure 5) reflected that the structured-sequence benefit was more pronounced in standard probes, $F(1,23)=25.65, p \leq .001, \eta_{p}^{2}=.527$, than in transfer probes, for which it was not significant, $F(1,23)=2.92$, $p \leq .101, \eta_{p}^{2}=.113$. In other words, participants responded faster during structured sequence runs than during random sequence runs only when the structured sequence was the one they had previously practiced with the same hand and not when it was the one they had

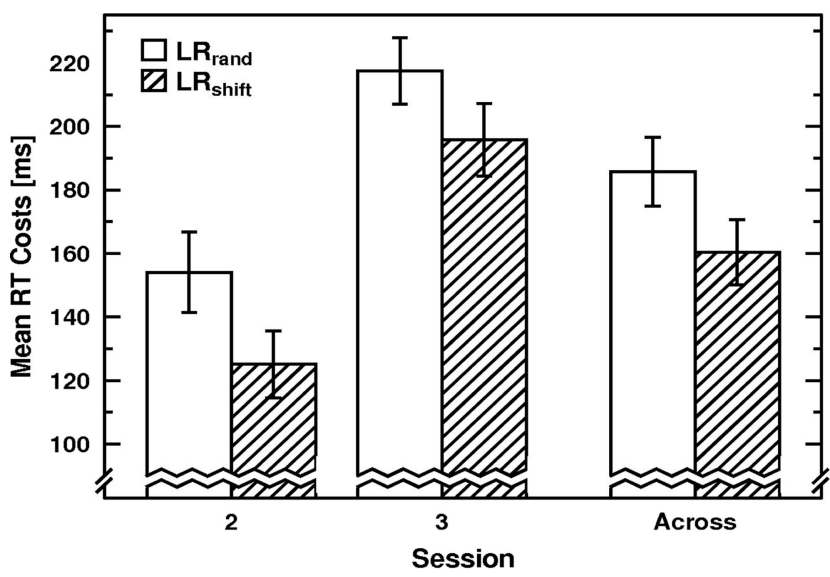

Figure 4. Mean reaction time (RT) costs ( \pm 1 SEM, respectively) separately for and across Sessions 2 and 3 in shift blocks in Experiment 2. $L R_{\text {rand }}=$ randomized block, both hands; $L R_{\text {shift }}=$ shift block, both hands.

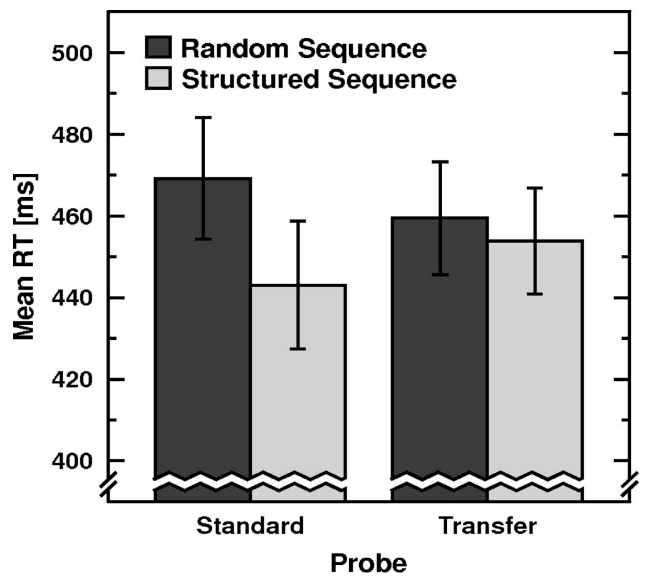

Figure 5. Mean reaction time (RT; \pm 1 SEM, respectively) in standard probes (structured sequence $=$ sequence practiced with the same hand during the bimanual task) and in transfer probes (structured sequence = sequence practiced with the other hand during the bimanual task) in the unimanual phase of Experiment 2.

previously practiced with the other hand. Mean error rates were computed and analyzed in an analogous manner, but the analysis yielded no significant effects, all $F_{\mathrm{s}}(1,23)<2.84$, all $p \mathrm{~s}>.105$, all $\eta_{p}^{2}<.110$.

\section{Discussion}

As in Experiment 1, participants extensively practiced an SRT task in which actions of both hands alternated and each hand performed its own fixed sequence of actions. Unlike Experiment 1, however, the hand-related sequences were correlated and thus established a relatively simple compound sequence. The occurrence of RT costs for both hands irrespective of whether only one or both of the two hand-related sequences were randomized indicates integrated learning of the compound sequence.

However, there was also evidence for partly independent learning of the hand-related sequences. For either hand it was the case that response speed deteriorated more pronouncedly when it lost its sequence while the other hand retained its sequence than when it was the other way around. This pattern suggests that, on top of massive integrated learning of the compound sequence, some degree of independent learning occurred for the hand-related constituent sequences. Evidence for partly independent learning also comes from shift probes. The shift probe yielded smaller RT costs than did concurrent randomization of both hand-related sequences. This shift probe advantage indicates that participants benefited from the within-hand regularities still present in the shift block even though between-hand regularities, which essentially define the compound sequence, were altered.

The independently acquired component of knowledge about the hand-related sequences appeared to be largely hand-specific. This finding conforms to the lack of intermanual transfer observed in Experiment 1, in which conditions for intermanual transfer were not as good as in Experiment 2.

An alternative explanation for the observed hand-specific enhancement of RT costs in terms of purely integrated learning of the compound sequence as discussed for Experiment 1 is not applica- 
ble here, because pseudorandom sequences were specifically selected so that there were no differences between the two hands in test blocks $L_{\text {rand }}$ and $R_{\text {rand }}$ in terms of deviations from the structured compound sequence (see Table 4). Moreover, the shift block advantage emerged although shift blocks contained more deviant tupels than did test blocks $L R_{\text {rand }}$.

As for the time course of integrated versus independent learning, integrated learning increased between Session 2 and Session 3, whereas there was no such improvement of independent learning. It is unclear whether this result reflects that independent learning had already fully developed by Session 2 or that independent learning develops more slowly than integrated learning and thus requires more practice to yield further improvements.

In summary, the results of Experiment 2 demonstrate that, in addition to the integrated learning of a compound sequence spanning both hands, the constituent hand-related sequences are learned partly independently of each other, even though the handrelated sequences were correlated and thus establish a relatively simple compound sequence. Moreover, the sequence knowledge that was acquired independently for the two hand-related sequences appears to be represented in a hand-specific manner.

\section{General Discussion}

Action sequences frequently involve multiple effectors. This is the case not only in a myriad of real-world tasks, such as buttoning a shirt, but also in laboratory tasks, such as the standard SRT task (e.g., Nissen \& Bullemer, 1987). Both example tasks require the coordinated use of the left and the right hand. Nevertheless, until now research has examined only integrated learning of an overall sequence spanning both hands and has neglected the possibility that at least partly independent learning of the hand-related constituent sequences might occur in such circumstances. The experiments reported here were designed to address this issue. In particular, we investigated the hypothesis that knowledge about the hand-related subsequences of a bimanual compound sequence might be acquired and represented partly independently of one another.

In two experiments we implemented a bimanual sequence for which actions of both hands alternated and each hand performed its own fixed sequence of actions. After extensive practice by participants, independent learning of the separate hand-related sequences was tested by randomizing (a) only one of the handrelated sequences and leaving the other intact or (b) both handrelated sequences in parallel.

In Experiment 1, performance decrements in randomization test blocks occurred primarily for responses executed with the hand that lost its sequence; performance with the other hand was comparatively spared. This pattern of results is consistent with partly independent learning of the two hand-related sequences. Indications for integrated learning of the compound sequence were weak. Because the compound sequence was established by merging two hand-related sequences of unequal length, the resulting compound sequence was considerably longer and more complex than the constituent hand-related sequences; this might have favored independent learning over integrated learning.

Therefore, in Experiment 2 we used equally long hand-related sequences, so that the resulting compound sequence was only twice as long as the constituent sequences and was not more complex in terms of statistical structure. Under these conditions, considerable integrated learning of the compound sequence was observed in randomization probes. However, on top of that, the hand-related sequences appeared to have been learned partly independently of each other. Further evidence for partly independent learning came from an additional test block in which the two hand-related sequences were shifted relative to each other, so that between-hands regularities were effectively destroyed and withinhand regularities were maintained. The occurrence of performance decrements in these shift probes reflects integrated learning. However, the finding that these shift costs were less pronounced than the costs associated with complete randomization of the compound sequence also indicates partly independent learning. Taken together, these findings suggest that practicing bimanual action sequences not only results in integrated learning of the overall sequence but, to some degree, in independent learning of the separate sequences pertaining to each hand.

Obviously, the task employed in the experiments reported here required some degree of bimanual coordination. Bimanual coordination has already been investigated extensively (for a review, see Swinnen \& Wenderoth, 2004). Much of this research was focused on determining whether and under which conditions bimanual tasks can be effectively controlled in an integrated fashion as a single task and when such integration breaks down. It has been demonstrated that adequate perceptual transformations and task conceptualizations can aid integration into a single task (e.g., Franz, Zelaznik, Swinnen, \& Walter, 2001; see also Mechsner, Kerzel, Knoblich, \& Prinz, 2001). Perceptually, the bimanual SRT task used here affords conceptualization as a single task because actions of both hands pertain to a single imperative stimulus, the position of which varies on a single spatial dimension. Still, the experiments reported here showed that hand-related sequences were learned at least partly independently. This finding might be taken to suggest that task integration was not complete. However, any comparisons between the present experiments and the substantial body of research on bimanual coordination are limited by the fact that the discrete keypressing task used here differs fundamentally from the more dynamic continuous tasks typically employed in research on bimanual coordination.

The issue of the nature of the memory representation underlying hand-related sequence knowledge was addressed in test blocks in which the sequence practiced with one hand was transferred to the other hand. Neither experiment yielded statistically significant evidence for intermanual transfer. This suggests that the independently acquired hand-related sequence knowledge is primarily hand-specific. This finding is consistent with evidence for the acquisition of effector-specific sequence knowledge from several other sources (Bapi et al., 2000; Jordan, 1995; Park \& Shea, 2003, 2005; Verwey \& Clegg, 2005; Verwey \& Wright, 2004; see also Berner \& Hoffmann, 2008, 2009) as well as with the model of Hikosaka et al. (1999), which postulates parallel neural networks for fast acquisition of effector-independent sequence knowledge in terms of visual-spatial coordinates and slow acquisition of effector-specific sequence knowledge in terms of motor coordinates. Also, the results reported here concur with the previous evidence for effector-specific sequence learning in suggesting that a strict modularity view of sequence representation (e.g., Keele et al, 1995) cannot be upheld. 
Furthermore, the present study demonstrates the concurrent acquisition of effector-specific sequence knowledge for two effectors (i.e., the two hands; see also Berner \& Hoffmann, 2008), which can certainly be accommodated within Hikosaka et al.'s (1999) model. Moreover, the findings from Experiment 1 suggest a specification of Hikosaka et al.'s model, insofar as they demonstrate the acquisition of hand-specific sequence knowledge even though learning of a sequence in terms of visual-spatial coordinates was largely absent. This further underscores the independence of the parallel levels of sequence representation postulated in Hikosaka et al.'s model. Previous research has demonstrated nontransferable, effector-specific sequence learning exclusively in addition to considerable transferable sequence learning. Experiment 1 suggests that effector-specific sequence knowledge can develop even when the overall sequence is too complex for much effector-independent learning to occur.

Effector-specific sequence learning has been suggested to pertain to the fine-tuning of sequence production to the biomechanical properties of the effector used (e.g.,Verwey \& Clegg, 2005; see also Jordan, 1995; Park \& Shea, 2005). This is equivalent to the development of coarticulation (cf. for the domain of speech production, Daniloff \& Moll, 1968; Kent \& Minifie, 1977), that is, the optimization of transitions between single movements in a sequence of movements. The view that such coarticulatory optimization is involved in hand-specific sequence learning receives some support from an experiment by Japikse et al. (2003). The sequence knowledge acquired by participants in this experiment was completely transferable from the practiced to the unpracticed hand, even after more than 1,000 sequence repetitions (an amount of sequence practice usually sufficient for the development of effector-specific sequence knowledge; e.g., Verwey \& Clegg, 2005). The sequence was four elements long and had a random element (r) inserted between any two sequence elements (e.g., ArDrBrCrArDrBrCr ....). The failure to acquire any hand-specific sequence knowledge under these conditions might have been due to the lack of a consistent sequence of response transitions for which the coarticulation of individual finger movements could have been optimized. Verwey and Clegg (2005) have argued that coarticulatory optimization plays a larger role for within-hand sequence execution than for between-hands sequence execution because biomechanical interactions among fingers of one hand are more severe than those between hands. This perspective suggests that independent learning of hand-related sequences occursdespite responses being executed alternately with the left and the right hand-because within-hand transitions are subject to coarticulatory optimization, whereas across-hands transitions would not be affected by the development of improved coarticulation. Even though this explanation remains highly speculative at this point, we find it quite compelling.

Finally, we would like to discuss the present findings in terms of a model formulated by Keele et al. (2003) that focuses on the interplay between integrated and independent sequence learning. Keele et al. proposed two subsystems: One is a unidimensional system encompassing independent learning modules, each of which is assumed to operate on input from only one dimension, so that independent sequence learning on any dimension takes place regardless of redundancies in other dimensions. Furthermore, a multidimensional system is assumed to enable integrated sequence learning by associating task-relevant (categorized) stimuli from different dimensions, provided that they are correlated and attended to. The model remains vague with regard to what exactly constitutes a dimension. As a first approximation, Keele et al. proposed that different modalities may constitute separate dimensions, but they noted that stimulus attributes within a modality might also constitute dimensions. Although they acknowledged uncertainties regarding the term dimension and the issue of exactly what constitutes a dimension, Keele et al. further speculated that "distinctions within the motor system (e.g., hands vs. feet) may also constitute dimensions" (p. 317) and, thus, establish independent sequence learning modules. Therefore, Keele et al.'s model can account for the integrated learning of the compound sequence established by correlated sequences in Experiment 2 as well as the relative lack of integrated learning of the compound sequence established by uncorrelated sequences in Experiment 1. Although it is certainly not necessary to invoke Keele et al.'s model-and the operation of the multidimensional system in particular-to explain the discrepancy between Experiments 1 and 2 in terms of evident integrated learning of the compound sequence, we believe this possibility at least deserves mention. Also, it is worth noting that Keele et al.'s model can account for concurrent effectorspecific sequence learning, insofar as it can accommodate the existence of separate sequence learning modules for different effectors (e.g., the two hands).

In conclusion, the findings reported here suggest that, after extensive practice, hand-specific sequencing skill develops concurrently but independently for the hand-related subsequences of a compound sequence spanning both hands not only when integrated learning of the bimanual sequence is weak but also when it dominates independent learning.

\section{References}

Bapi, R. S., Doya, K., \& Harner, A. M. (2000). Evidence for effector independent and dependent representations and their differential time course of acquisition during motor sequence learning. Experimental Brain Research, 132, 149-162.

Berner, M. P., \& Hoffmann, J. (2008). Effector-related sequence learning in bimanual-bisequential serial reaction time task. Psychological Research, 72, 138-154.

Berner, M. P., \& Hoffmann, J. (2009). Acquisition of effector-specific and effector-independent components of sequencing skill. Journal of Motor Behavior, 41, 30-44.

Clegg, B. A. (2005). Stimulus-specific sequence representation in serial reaction time tasks. Quarterly Journal of Experimental Psychology, 58(A), 1087-1101.

Clegg, B. A., DiGirolamo, G. J., \& Keele, S. W. (1998). Sequence learning. Trends in Cognitive Science, 2, 275-281.

Cohen, A., Ivry, R. I., \& Keele, S. W. (1990). Attention and structure in sequence learning. Journal of Experimental Psychology: Learning, Memory, and Cognition, 16, 17-30.

Daniloff, R., \& Moll, K. (1968). Coarticulation of lip rounding. Journal of Speech and Hearing Research, 11, 707-721.

Dean, N. J., Kovacs, A. K., \& Shea, C. H. (2008). Transfer of movement sequences: Bigger is better. Acta Psychologica, 127, 355-368.

Deroost, N., \& Soetens, E. (2006). Perceputual or motor learning in SRT tasks with complex sequence structures. Psychological Research, 70, 88-102.

Deroost, N., Zeeuws, \& Soetens, E. (2006). Effector-dependent and response location learning of probabilistic sequences in serial reaction time tasks. Experimental Brain Research, 171, 469-480. 
Deroost, N., Zeischka, P., \& Soetens, E. (2008). Negative priming in the SRT task: Learning of irrelevant sequences is enhanced by concurrent learning of relevant sequences. European Journal of Cognitive Psychology, 20, 47-68.

Franz, E. A., Zelaznik, H. N., Swinnen, S., \& Walter, C. (2001). Spatial conceptual influences on the coordination of bimanual actions: When a dual task becomes a single task. Journal of Motor Behavior, 33, 103112

Frensch, P. A., \& Miner, C. S. (1995). Zur Rolle des Arbeitsgedächtnisses beim impliziten Sequenzlernen [The role of working memory in implicit sequence learning]. Zeitschrift für Experimentelle Psychologie, 42, 545575 .

Grafton, S. T., Hazeltine, E., \& Ivry, R. B. (1998). Abstract and effectorspecific representations of motor sequences identified with PET. Journal of Neuroscience, 18, 9420-9428.

Grafton, S. T., Hazeltine, E., \& Ivry, R. B. (2002). Motor sequence learning with the nondominant left hand. Experimental Brain Research, 146, 369-378.

Hazeltine, E. (2002). The representational nature of sequence learning: Evidence for goal-based codes. In W. Prinz \& B. Hommel (Eds.), Common mechanisms in perception and action (pp. 675-689). New York: Oxford University Press.

Hikosaka, O., Nakahara, H., Rand, M. K., Sakai, K., Lu, X., Nakamura, K., et al. (1999). Parallel neural networks for learning sequential procedures. Trends in Neuroscience, 22, 464-471.

Hikosaka, O., Nakamura, K., Sakai, K., \& Nakahara, H. (2002). Central mechanisms of motor skill learning. Current Opinion in Neurobiology, 12, 217-222.

Hoffmann, J. (2001). Cognitive psychology of representation of serial order. In N. Smelser \& P. Baltes (Eds.), International encyclopedia of the social and behavioral sciences (Vol. 19, pp. 13171-13174). Oxford, England: Elsevier.

Hoffmann, J., \& Koch, I. (1998). Implicit learning of loosely defined structures. In M. A. Stadler \& P. Frensch (Eds.), Handbook of implicit learning (pp. 161-199). Thousand Oaks, CA: Sage.

Hoffmann, J., Martin, C., \& Schilling, A. (2003). Unique transitions between stimuli and responses in SRT tasks: Evidence for the primacy of response predictions. Psychological Research, 67, 160-173.

Hoffmann, J., \& Sebald, A. (1996). Reiz- und Reaktionsmuster in seriellen Wahlreaktionen [Stimulus and response patterns in serial choice reactions]. Zeitschrift für experimentelle Psychologie, 63, 40-68.

Hoffmann, J., Sebald, A., \& Stöcker, C. (2001). Irrelevant response effects improve serial learning in serial reaction time tasks. Journal of Experimental Psychology: Learning, Memory, and Cognition, 27, 470-482.

Howard, D. V., Howard, J. H., Jr., Japikse, K., DiYanni, C., Thompson, A., \& Somberg, R. (2004). Implicit sequence learning: Effects of level of structure, adult age, and extended practice. Psychology and Aging, 19, $79-92$.

Howard, J. H., Jr., \& Howard, D. V. (1997). Age differences in implicit learning of higher order dependencies in serial patterns. Psychology and Aging, 12, 634-656.

Howard, J. H., Jr., Howard, D. V., Dennis, N. A., Yankovich, H., \& Vaidya, C. J. (2004). Implicit spatial contextual learning in healthy aging. Neuropsychology, 18, 124-134.

Japikse, K. C., Negash, S., Howard, J. H., Jr., \& Howard, D. V. (2003). Intermanual transfer of procedural learning after extended practice of probabilistic sequences. Experimental Brain Research, 148, 38-49.

Jiménez, L., Vaquero, J. M. M., \& Lupiáñez, J. (2006). Qualitative differences between implicit and explicit sequence learning. Journal of Experimental Psychology: Learning, Memory, and Cognition, 32, 475490.

Jordan, M. I. (1995). The organization of action sequences: Evidence from a relearning task. Journal of Motor Behavior, 27, 179-192.

Keele, S. W., Ivry, R., Mayr, U., Hazeltine, E., \& Heuer, H. (2003). The cognitive and neural architecture of sequence representation. Psychological Review, 110, 316-339.

Keele, S. W., Jennings, P., Jones, S., Caulton, D., \& Cohen, A. (1995). On the modularity of sequence representation. Journal of Motor Behavior, 27, 17-30

Kent, R. D., \& Minifie, F. D. (1977). Coarticulation in recent speech production models. Journal of Phonetics, 5, 115-133.

Kinder, A., Rolfs, M., \& Kliegl, R. (2008). Sequence learning at optimal stimulus-response mapping: Evidence from a serial reaction time task. Quarterly Journal of Experimental Psychology, 61, 203-209.

Koch, I., \& Hoffmann, J. (2000a). The role of stimulus-based and response-based spatial information in sequence learning. Journal of Experimental Psychology: Learning, Memory, and Cognition, 26, 863882

Koch, I., \& Hoffmann, J. (2000b). Patterns, chunks, and hierarchies in serial reaction-time tasks. Psychological Research, 63, 22-35.

Lashley, K. S. (1951). The problem of serial order in behavior. In L. A Jeffress (Ed.), Cerebral mechanisms in behavior: The Hixon symposium (pp. 112-136). New York: Wiley.

Marcus, D. J., Karatekin, C., \& Markiewicz, S. (2006). Oculomotor evidence of sequence learning on the serial reaction time task. Memory \& Cognition, 34, 420-432.

Mayr, U. (1996). Spatial attention and implicit sequence learning: Evidence for independent learning of spatial and nonspatial sequences. Journal of Experimental Psychology: Learning, Memory, and Cognition, 22, 350-364.

Mechsner, F., Kerzel, D., Knoblich, G., \& Prinz, W. (2001). Perceptual basis of bimanual coordination. Nature, 414, 69-73.

Muehlbauer, T., Panzer, S., \& Shea, C. H. (2007). The transfer of movement sequences: Effects of decreased and increased load. Quarterly Journal of Experimental Psychology, 60, 770-778.

Nattkemper, D., \& Prinz, W. (1997). Stimulus and response anticipation in a serial reaction task. Psychological Research, 60, 98-112.

Nissen, M. J., \& Bullemer, P. (1987). Attentional requirements of learning: Evidence from performance measures. Cognitive Psychology, 19, 1-32.

Panzer, S., Büsch, D., Shea, C. H., Mühlbauer, T., Naundorf, F., \& Krüger, M. (2007). Dominanz visuell-räumlicher Codierung beim Lernen von Bewegungssequenzen [Movement sequence learning and the dominance of visual-spatial coding]. Zeitschrift für Sportpsychologie, 14, 123-129.

Park, J.-H., \& Shea, C. H. (2003). Effect of practice on effector independence. Journal of Motor Behavior, 35, 33-40.

Park, J.-H., \& Shea, C. H. (2005). Sequence learning: Response structure and effector transfer. Quarterly Journal of Experimental Psychology, 58(A), 387-419.

Remillard, G. (2003). Pure perceptual-based sequence learning. Journal of Experimental Psychology: Learning, Memory, and Cognition, 29, 581597.

Remillard, G. (2008). Implicit learning of second-, third-, and fourth-order adjacent and nonadjacent sequential dependencies. Quarterly Journal of Experimental Psychology, 61, 400-424.

Remillard, G., \& Clark, J. M. (2001). Implicit learning of first-, second-, and third-order transition probabilities. Journal of Experimental Psychology: Learning, Memory, and Cognition, 27, 483-498.

Rhodes, B. J., Bullock, D., Verwey, W. B., Averbeck, B. B., \& Page, M. P. A. (2004). Learning and production of movement sequences: Behavioral, neurophysiological, and modelling perspectives. Human Movement Science, 23, 699-746.

Riedel, B., \& Burton, A. M. (2006). Auditory sequence learning: Differential sensitivity to task relevant and task irrelevant sequences. Psychological Research, 70, 337-344.

Rüsseler, J., Münte, T. F., \& Rösler, F. (2002). Influence of stimulus distance in implicit learning of spatial and nonspatial event sequences. Perceptual and Motor Skills, 95, 973-987.

Schmidtke, V., \& Heuer, H. (1997). Task integration as a factor in 
secondary-task effects on sequence learning. Psychological Research, $60,53-71$.

Schneider, W., Eschman, A., \& Zuccolotto, A. (2002). E-Prime user's guide. Pittsburgh, PA: Psychology Software Tools.

Stöcker, C., \& Hoffmann, J. (2004). The ideomotor principle and motor sequence acquisition: Tone effects facilitate movement chunking. Psychological Research, 68, 126-137.

Stöcker, C., Sebald, A., \& Hoffmann, J. (2003). The influence of responseeffect compatibility in a serial reaction time task. Quarterly Journal of Experimental Psychology, 56(A), 586-703.

Stadler, M. A. (1989). On learning complex procedural knowledge. Journal of Experimental Psychology: Learning, Memory, and Cognition, 15, 1061-1069.

Swinnen, S. P., \& Wenderoth, N. (2004). Two hands, one brain: Cognitive neuroscience of bimanual skill. Trends in Cognitive Sciences, 8, 18-25.

Verwey, W. B., \& Clegg, B. A. (2005). Effector dependent sequence learning in the serial RT task. Psychological Research, 69, 242-251.

Verwey, W. B., \& Wright, D. L. (2004). Effector-independent and effectordependent learning in the discrete sequence production task. Psychological Research, 68, 64-70.

Wilde, H., \& Shea, C. H. (2006). Proportional and nonproportional transfer of movement sequences. Quarterly Journal of Experimental Psychology, $59,1626-1647$.

Willingham, D. B. (1999). Implicit motor sequence learning is not purely perceptual. Memory \& Cognition, 27, 561-572.

Willingham, D. B., Wells, L. A., Farrell, J. M., \& Stemwedel, M. E. (2000). Implicit motor sequence learning is represented in response locations. Memory \& Cognition, 28, 366-375.

Witt, J. K., \& Willingham, D. T. (2006). Evidence for separate representations for action and location in implicit motor sequencing. Psychonomic Bulletin \& Review, 13, 902-907.

Ziessler, M. (1998). Response-effect learning as a major component of implicit serial learning. Journal of Experimental Psychology: Learning, Memory, and Cognition, 24, 962-978.

Ziessler, M., \& Nattkemper, D. (2001). Learning of event sequences is based on response-effect learning: Further evidence from a serial reaction task. Journal of Experimental Psychology: Learning, Memory, and Cognition, 27, 595-613.

Received July 23, 2007

Revision received January 9, 2009

Accepted January 13, 2009 